\title{
Learner-User Satisfaction Survey in the AdaptWeb Platform using the Learner Choices from Learner-Driven Learning
}

\author{
Alessandro da Silveira Dias \\ Universidade Federal do Rio Grande do Sul \\ alessandrosdias@gmail.com
}

\author{
Leandro Krug Wives \\ Universidade Federal do Rio Grande do Sul \\ leandro.wives@ufrgs.br
}

\begin{abstract}
In this paper, we review the definition of the learner choices from the Learner-driven Learning paradigm for e-learning systems. After this, we analyze how different categories of e-learning systems enable the user to make these choices, such as Serious Games. We present in detail how AdaptWeb platform makes available these choices to learner users. Additionally, we present a satisfaction survey performed after an online course on AdaptWeb platform. The survey questions were about making choices during learning and about the way AdaptWeb makes the choices available to learner-users. Summarizing the results, students enjoyed being able to make choices about their own learning and felt that this possibility was beneficial to their learning. Moreover, they liked the way AdaptWeb makes the choices available to students. Most of the students found the system easy to use, intuitive, and the student's choices were explicit and easy to take.
\end{abstract}

Keywords: Learner-driven Learning; Ubiquitous e-Learning Systems; AdaptWeb; Satisfaction Survey 


\section{Introduction}

During a systematic review on recommender systems of learning objects (LOs) we noticed that learner-users usually perform a set of choices or make decisions during learning (e.g., "what to learn", "how to learn", "with whom to learn”, "in which pathway to learn”, "where to learn", among others), depending on the platform used to learn. This set of choices belongs to the Learner-driven Learning paradigm from Education (Alexander et al., 2004; Watkins et al., 2007).

The Learner-driven Learning paradigm focuses on the process by which the learner takes control of his own learning, in particular, how he sets his own learning goals, how he finds the appropriate resources, how he decides what learning methods he uses, and how he assesses his progress (Alexander et al., 2004). It is interrelated with other terms of Education and Psychology, such as autonomous learning, responsible learning, self-regulated learning, and active learning.

In a previous paper (Dias \& Wives, 2018), we reviewed the origin of the Learner-driven Learning paradigm: the Student-centered Learning paradigm and analyzed this learning paradigm from the point of view of formal and non-formal learning environments. In both cases, the learner performs choices during the learning process. We have found that the set of choices from the Learner-driven Learning are presented in a more general way in Alexander et al. (2004), Miliband (2006), Watkins et al. (2007), LEADLAB (2010), Ginsberg (2015), and others. Then we defined these choices for e-learning systems, based on user-interaction patterns and navigational patterns that we have noticed while we knew different e-learning systems during the systematic review performed. To the best of our knowledge, we were the first to specify these choices for e-learning systems.

We also have analyzed how these three categories of e-learning systems Massive Open Online Courses (MOOCs), Online Discovery Learning Spaces (ODLSs), and Personal Learning Environments (PLEs) enable users to perform such choices. Additionally, we presented the user choices learners have in the last version of the AdaptWeb ${ }^{1}$ platform, a ubiquitous e-learning system for formal education. For illustration, we created an online course of Interaction Diagrams of UML on the AdaptWeb to show how the learner-user performs such choices.

In the current paper, that is an extension of (Dias \& Wives, 2018), we review (I) the definition of the learner choices from the Learner-driven Learning paradigm for e-learning systems, and (II) these choices on the AdaptWeb platform, now with more details. The goal is to present an online satisfaction survey performed after the online course of Interaction Diagrams of UML on the AdaptWeb platform with learner choices, which was conducted among their participants.

There were 45 subjects. This survey was based on open-ended questions. The advantage of this type of survey questions, over closed-ended questions, is that subjects can respond to the questions exactly as how they would like to answer them, it is, they do not only choose among generic response alternatives (Reja et al., 2003). The survey questions were about making choices during learning and about the way AdaptWeb makes the choices available to learnerusers. Summarizing the results, students enjoyed being able to make choices about their own learning and felt that this possibility was beneficial to their learning. And they liked the way

\footnotetext{
${ }^{1}$ http://adaptweb.sourceforge.net/
} 
AdaptWeb makes the choices available to students. Most of the students found the system easy to use, intuitive, and the student's choices were explicit and easy to take.

The rest of this paper is organized as follows: Section 2 gives a brief overview of related works. Section 3 gives a brief review of the definition of learner choices from the Learnerdriven Learning for e-learning Systems and also analyzes more categories of e-learning systems that enable the user to perform these choices, such as, Serious Games. Section 4 presents in detail such learner-user choices in the AdaptWeb platform and briefly presents how to use such choices for the personalization of learning, by a recommender system of learning objects (LOs). Section 5 presents the learner-user satisfaction survey performed. Finally, Section 6 presents our conclusions.

\section{Related Work}

As previously mentioned, to the best of our knowledge, we were the first to specify the choices from the Learner-driven Learning paradigm of learners for e-learning systems. It is ensured by the systematic review we performed. Therefore there is no way to compare our work side by side with existing work. Then we present bellow intersections between our research and existing work.

We organized the related work into two groups: Relation between Personalized Learning and the Choices from the Learner-driven Learning Paradigm, and the Relation between Recommender Systems and the Choices from the Learner-driven Learning Paradigm.

\subsection{Relation between the term "Personalized Learning" and the Choices from the Learner-driven Learning Paradigm}

Many works use the choices from the Learner-driven Learning paradigm, such as "what to learn" and "how to learn", to define the term "personalized learning". For example: according to Gates-Dell-EDUCAUSE (2017) personalized learning seeks to accelerate student learning by tailoring the instructional environment - "what", "when", "how", and "where" students learnto address the individual needs skills and interests of each student. Students can take ownership of their own learning, while also developing deep, personal connections with each other, their teachers and other adults. Other example, according to iNACOL (2013) personalized learning is tailoring learning for each student's strengths, needs, and interests - including enabling student voice and choice in "what", "how", "when" and "where" they learn - to provide flexibility and supports to ensure mastery of the highest standards possible.

Song (2017) investigates the elements for realizing personalized learning and to figure out whether they have been applied to MOOCs. This research focused on the MOOCs learners' experience about personalized learning. A quantitative questionnaire was applied as the main method for collecting data. Authors did not study all the factors for personalized learning on MOOCs, but rather a subset of factors, such as learning styles and adaptive instruction, to test the relevant factors. Therefore a quantitative method was more suitable. In the questionnaire, there were questions related to these choices from the Learner-driven Learning: "what to learn", "how to learn”, "when to learn”, "where to learn”, "with whom to learn”, and "how to assess learning”.

Other works, such as Mackness et al. (2010) and Downes (2008), also present relations between "Personalized Learning" and the choices from the Learner-driven Learning Paradigm.

Downes (2008) has suggested that the key characteristics of an online course using connectivist principles are Autonomy, Diversity, Openness, and Connectedness, and 
Interactivity. It presents these four concepts, where autonomy means: to allow learners maximum choice of "where", "when”, "how", "with whom” and even "what" to learn.

Based on this, Mackness et al. (2010) present findings of research which explored learner experiences in a MOOC course that applies these four connectivist characteristics. It had more than 2000 learners around the world. In this MOOC course, learners were encouraged to be autonomous in their choice of technology for interaction on the course and ways of working. A variety of course sites were used, such as Moodle forums, Ustream (video streaming), Elluminate (web conferencing), Facebook, and Second Life (which refers to "where to learn" and "when to learn" to learn choices). The course attracted a diverse group of participants from around the world and whilst English was the dominant language, some participants established non-English speaking groups, such as Spanish ("with whom to learn" choices). The course made use of readings and presentations already freely available on the Web, and these were supplemented by course instructor and visiting speaker presentations and materials ("what to learn" and "how to learn" choices). The course content was available from the start on the course wiki, and participants were free to plan their own paths through this content ("in which pathway to learn” choices).

Another relevant work is the one of McClaskey (2016), which discusses how teachers can develop learners to become expert learners. In personalized learning environments, learners develop skills to become self-directed learners. The author discusses the stages of personalized learning environments: Stage One Teacher-centered, Stage Two Learner-centered, and Stage Three Learner-driven; and explains the types of choices at each stage. All kinds of choices can be done. And all stages can be supported by technological tools.

In Stage One Teacher-centered, the teacher offers the learner choices to access content through images, videos, text-based resources, audio, hands-on activities, or interactions with peers. The learner demonstrates their strengths. As the learner moves to Stage Two LearnerCentered, as a co-designer, the teacher guides the learner then gets out of the way as learners make choices in their learning. The teacher collaborates with the learner to brainstorm ideas for lesson design, strategies for peer and self-assessment, types of tools and resources to use with activities, etc. During Stage Two, the learner chooses topics and direction for what they plan to design based on personal interests and questions generated individually or with peers. In Stage Three Learner-Driven, the learners are an expert learner. They choose a challenge or problem that they are passionate about and soon they discover their purpose for learning.

\subsection{Relation between Recommender Systems and the Choices from the Learner-driven Learning Paradigm}

Drachsler et al. (2015) present a review of recommender systems in Technology Enhanced Learning. Recommender systems are filtering systems that usually reduce a considerable number of options in a user choice to a smaller subset and then leave it to the user to select an option from the subset. This work presents different recommendation tasks, such as finding good LOs, finding peer learners, and recommending learning pathways. These three recommendation tasks are related to these learner choices: "what to learn", "with whom to learn", and "in which learning pathway to learn". Therefore, when learner-users have many options in a choice, a recommender system can help them deal with the overload of options.

Zheng et al. (2014) formalized the recommendation task of "context suggestion". Recommenders systems of context are applied, for instance, to maximize user experience, which refers to a person's emotions and attitudes about using a particular product, system, or service. For instance, a recommender system that performs this recommendation task can identify the best contexts (place, time and/or companion) for a learner-user to learn some content. In this best context, for instance, learning might be enhanced. Recommendation of places, times and 
companions are related to the following learner choices: "where to learn”, "when to learn” and "with whom to learn".

Zhuhadar and Butterfield (2014) launch the idea of an LO recommendation approach for MOOCs based on the continued monitored user interactions into all stages of course, in the learning performance and the previous knowledge of the user. We see that this approach includes all choices of the Learner-driven Learning indirectly, that is, as previously described in Mackness et al. (2010) where we describe the learner's actions and place in parentheses the respective choices from the Learner-Driven Learning paradigm.

Finally, Intayoad et al. (2020) propose a method to provide personalized recommendation for online learning systems that guides learners to the right LOs at the right time. This method is based on the contextual bandits and reinforcement learning problems. It uses the past student behaviors and current student state as the contextual information to create the policy for the reinforcement agent to make the optimal decision. This method uses the past LOs chosen by learners and their learning pathways. These information are related to these learner choices "what to learn", "how to learn”, and "in which learning pathway to learn".

\section{Learner-Driven Learning in E-learning Systems}

In this section we briefly review the definition of learner choices from Learner-driven Learning for e-learning Systems, presented in our previous paper (Dias \& Wives, 2018). In this, we analyzed how these three categories of e-learning systems Massive Open Online Courses (MOOCs), Online Discovery Learning Spaces (ODLSs), and Personal Learning Environments (PLEs) enable users to perform such choices. Now we extend this analysis, showing how other categories of e-learning systems, such as, Serious Games and Adaptive Learning Systems, enable the user to perform such choices.

\subsection{Definition of the Learner Choices for E-learning Systems}

The set of choices from the Learner-driven Learning paradigm are presented in a more general way in Alexander et al. (2004), Miliband (2006), Watkins et al. (2007), LEADLAB (2010), Ginsberg (2015), and others. This general definition is always directed to some context. For instance, the definition of "what to learn" for formal learning environments is: for Passe (1996): "it is the student to choose disciplines, among the available ones, and thus he/she assembles a customized program of the course"; for Watkins (2007): "there are several student choices within the classroom, such as - which parts of a text should I read?, which exercises in a list should I do?, etc.”.

We presented a definition of these choices for the context of e-learning systems. We made this based on user-interaction patterns and navigational patterns we noticed while we knew different e-learning systems during a systematic review, as follows.

A choice of type what to learn means the topic of knowledge a user-preferred or wanted to learn. For instance, when the user is learning UML, this user has different diagrams to learn, e.g., Sequence diagram, Class diagram, Timing Diagrams, Activities diagram, etc. Ahead of these options, let's suppose the user chooses the Timing Diagrams to learn.

The sequence of topics over time the user preferred to follow to learn results in an upperlevel type of choice in which learning pathway to learning from. For instance, when the user is learning UML and prefers to start learning the Timing Diagrams, then this user may choose the Sequence Diagram, then the Activities Diagram, and so on. 
A choice of type how to learn specifies what LOs a user-preferred to use to learn a topic. For example, a subject can be learned using hypertext, a simulator, a video following a deductive learning strategy, or an inductive learning strategy; let's say that ahead these options, the user chooses the simulator to learn.

Choices of the type where to learn and when to learn mean, respectively, the geographic place and the time the user preferred to use to learn. For instance, the user chose to learn on Tuesday at home, and Friday at the lab.

Choices of the type with whom to learn mean the people the user interacted to learn a topic. For instance, in MOOCs, as it is common to have thousands of students, some MOOCs have a team of tutors, others have none or a very small team in comparacion the the number of students. Thus, when students do not understand content using the available LOs, they seek another user in the system to learn from.

Choices of type how much to learn mean the amount of knowledge the user sought to learn of a topic. It can be measured in closed-corpus applications (Manouselis et al. 2010), in this case, e-learning systems designed for formal education, i.e., those where the learning environment, learning resources, learning pathways, and assessment procedures are structured and receive maintenance. Thus, it should be possible to measure the amount of knowledge the user sought to learn of a topic. For example, consider a topic divided into 5 parts. The leaner user can seek to learn only 2 parts, since the other parts he already knows or does not find relevant to learn.

In the case of social learning networks, these are open-corpus applications (Manouselis et al. 2010), i.e., applications with the absence of structure and maintenance. In these, as knowledge increases continuously by the user community, there is no way to measure knowledge for a topic.

Choices of type how to assess learning mean how users measured their knowledge of a topic. For instance, in an e-learning system, there are different ways to assess the user's learning: quick online test, bibliographical research, audio-visual presentation, teamwork implementation project, through dialectics, presenting e-portfolios, and so on. For instance, ahead of these options, the user preferred to be assessed by an audio-visual presentation.

\subsection{The Learner Choices in Categories of E-learning Systems}

There are different categories (or models) of e-learning systems, such as Personal Learning Environments (PLEs), Massive Open Online Courses (MOOCs), Serious Games, etc. Some of them are presented below, with the choices that they usually make available to the learner-user.

Computer-based Trainings: these learning environments became popular in the 1990s, where courses and training were mainly provided on CD-ROMs, and people could access them from their personal computers. In these environments, learning is individual and involves interactions with multimedia material, such as audio and video. In systems of this model, learner-users generally choose which subjects they learn ("what to learn") and "in which learning pathway to learn" such subjects. The learner can choose "when to learn" and "where to learn", as they can carry the system and perform learning on computers from different locations, such as home and work. These systems usually provide resources for self-assessment ("how to assess learning”).

Web-based Trainings: correspond to online courses. Through the Internet, courses and training are offered online. It is an extension of the previous model, allowing new learning possibilities ("with whom to learn” choices), such as, collective learning in forums within the system, questioning through chats with teachers/tutors of the course, etc. 
Adaptive Learning Systems: it is an extension of the previous models; correspond to learning environments where computers help the learning process actively, with the goal of better meeting the unique needs of each learner. This help can occur in several ways. For example, in Web-based Training, using dynamic pages, the system can modify the way that the learning resources are delivered, based on the user profile and/or learning context. Computerbased Training can make knowledge-based adaptation, for example, if a learner is taking too long to complete a learning activity, the system suggests an easier one that will help the learner to complete the current activity.

In Section 4, we present the AdaptWeb platform, a ubiquitous e-learning system for formal education, which is an adaptive learning system. The last version of it allows the learner-user to make different choices: "what to learn”, "with whom to learn”, "how to learn”, "where to learn”, "when to learn", and "in which pathway to learn" during learning. This system is linked internally to a LO recommender system that uses the result of the user choices as a source of information.

Online Cooperative Learning Systems: systems in this model follow a pedagogical approach: Computer-supported Collaborative Learning. It corresponds to learning through social interactions, mainly through systems on the Internet. Learning is characterized by sharing and building knowledge among people using computing and communication technologies. In systems of this model, the learner often makes choices about "with whom to learn", "when to learn", and "where to learn".

Serious Games: correspond to learning within games. A serious game is a software and/or hardware developed through the principles of interactive game design with the goal of delivering educational content or user training. It is projected more for educational purposes than for entertainment. Learning is usually self-directed; however, it can also be adaptive or collaborative. In systems of this model, the learner can choose, for example, "where to learn", and "when to learn", and also "with whom to learn" on cooperative serious games.

Online Discovery Learning Spaces: correspond to learning environments that allow an active learning experience and self-directed by the learner. In systems of this model, learnerusers construct their knowledge through the exploration of LOs, that is, they make choices about "what to learn", "in which pathway to learn" and "how to learn". If they are available on the Internet, users can choose "when to learn" and "where to learn". Some systems provide LOs for self-assessment ("how to assess learning"). If the system is a social learning network, users can seek other users for peer learning ("with whom to learn”).

Personal Learning Environments: learning is a lifelong learning process, that is, it is continuous, voluntary, self-motivated and self-directed to meet personal and professional needs. To support this, systems where the learner can create their own learning goals and control the management of their own lifelong learning emerged. Such systems often involve Web 2.0 services, such as social networking and online discovery learning spaces. In systems of this model, generally, all kinds of choices are observed: “what to learn”, “with whom to learn”, etc.

Massive Open Online Courses: correspond to open, free and accessible online courses for a large simultaneous audience. These courses are generally for higher education and provided by renowned universities. In this model, the learner usually makes choices about "when to learn" and "where to learn", and "with whom to learn" when the MOOC does not have a team of tutors and the learner cannot learn alone only using the available LOs.

As it turns out, regardless of the category of e-learning system, the user always has choices to make during learning. Now that learning choices have been defined for e-learning systems, theses systems can be analyzed from this perspective. For example, an educational institution 
might wonder "which of these choices could I include in our MOOC to add value to it? and thus better serve learners."

Another example, the e-learning system of an educational institution can use the results of the learner-user choices to better understand their preferences, and thus better meet their needs during learning in an automated manner. In the next section, we will look at an example of this, where the results of these choices are used as the source of information for an LO recommendation system.

\section{Learner-User Choices from Learner-driven Learning in the AdaptWeb Platform}

In our previous paper (Dias \& Wives, 2018), we presented, briefly, the learner-user choices from Learner-driven Learning in the last version of the AdaptWeb platform. In this paper, we extend this presentation by putting more screens of the system and by showing details about the recommender system of LOs internally linked to the AdaptWeb.

We use an online course of Interaction Diagrams of UML in the AdaptWeb to illustrate how the learner-user performs such choices. This system allows the user to perform almost all types of choices from the Learner-driven Learning during learning.

\subsection{Learning Process in the AdaptWeb Platform}

In the latest version of the AdaptWeb, the creation and structuring of online courses occur as follows. The teacher performs such tasks through an authorship tool of the system. Firstly, the teacher creates an online course and defines the set of topics. Then, for each topic, the teacher informs metadata (title, description, category, etc.), determines prerequisites, associates a set of LOs from a repository to each topic, and creates an online test. The LOs have metadata that follow the IEEE LOM standard. After creating and structuring the course, the teacher publishes the course.

In general, the learning process in AdaptWeb occurs as follows: it has a beginning point; a learning cycle, where the learner-user learns one topic of the course by cycle; and a finish point. This process is detailed below.

When the user (the student) begins the course, the system presents an introductory text, and the user can choose complementary topics to learn, optionally with teacher's help, according to her personal preferences and needs. This is "what to learn" choice. With this, the user builds a customized program course, composed of the mandatory topics and the chosen complementary topics. For illustration, Figure 1 shows part of the screen where the user can choose complementary topics to learn, that is, where he/she performs the "what to learn" choice.

After performing this choice, the learning cycle starts. During the progress of the course, the system asks the user "which of the following topics do you want to learn from now on?" and provides the topics available for them to learn, according to the topics they have already completed, and to the prerequisites not yet learned. For each available topic, a brief overview is presented. It is illustrated in Figure 2; in the current moment, the learner-user must choose the next topic to start to learn: Communication diagram, Sequence diagram or Times diagrams. 


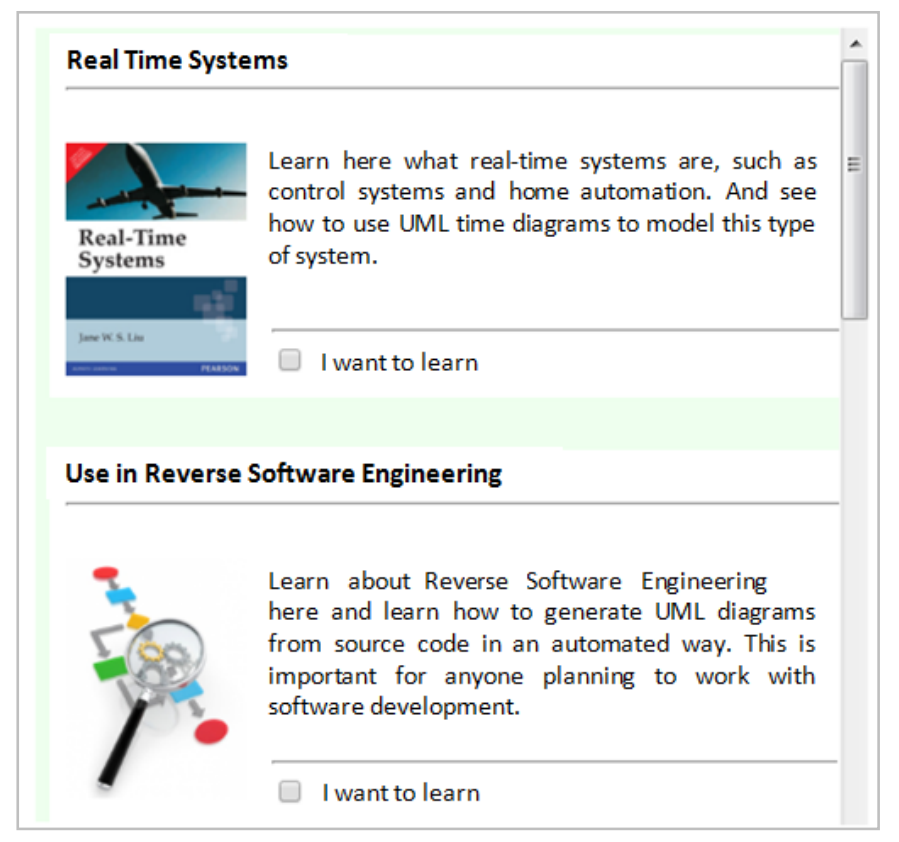

Figure 1: Part of the screen of AdaptWeb where the user performs the "what to learn" choice.

The sequence of choices about the next topic to learn gives rise to the users' learning pathway into the course. The sequence of topics the user preferred to follow to learn over time results in the upper-level type of choice "in which learning pathway to learn". It is a type of user trace into the system, a learning trace.

Within each topic of the course, the user can learn its content in different ways, that is, using different LOs according to his/her learning preferences and context restrictions. Each topic to be learned is associated with several LOs, from the LO repository. Figure 3 presents the screen of topics, where the user performs "how to learn" choices.

Figure 3 presents the moment in which the user is learning the topic Timing Diagrams of UML. This topic has 12 LOs to learn from; they are presented in the list on the left side. When the user selects an LO, the system displays the most relevant metadata (Dias \& Wives, 2018-b). The user uses this information to compare and choose LOs to use to learn. Using the link "Use this", he/she accesses the LO to learn, or to quickly check the LO inside, before taking the final decision for what LO to use.

In relation to "with whom to learn", AdaptWeb has modules for performing collaboration and communication. There is a forum where the user can create a topic about a subject, and then, all the users can discuss the matter. Moreover, users can exchange private text messages to each other, for instance, one user sends a message seeking to learn content from another user (a "with whom to learn" choice). In relation to "where to learn” and "when to learn”, these choices correspond to the place and time the user uses the system. These are recorded by the system as contextual information. 


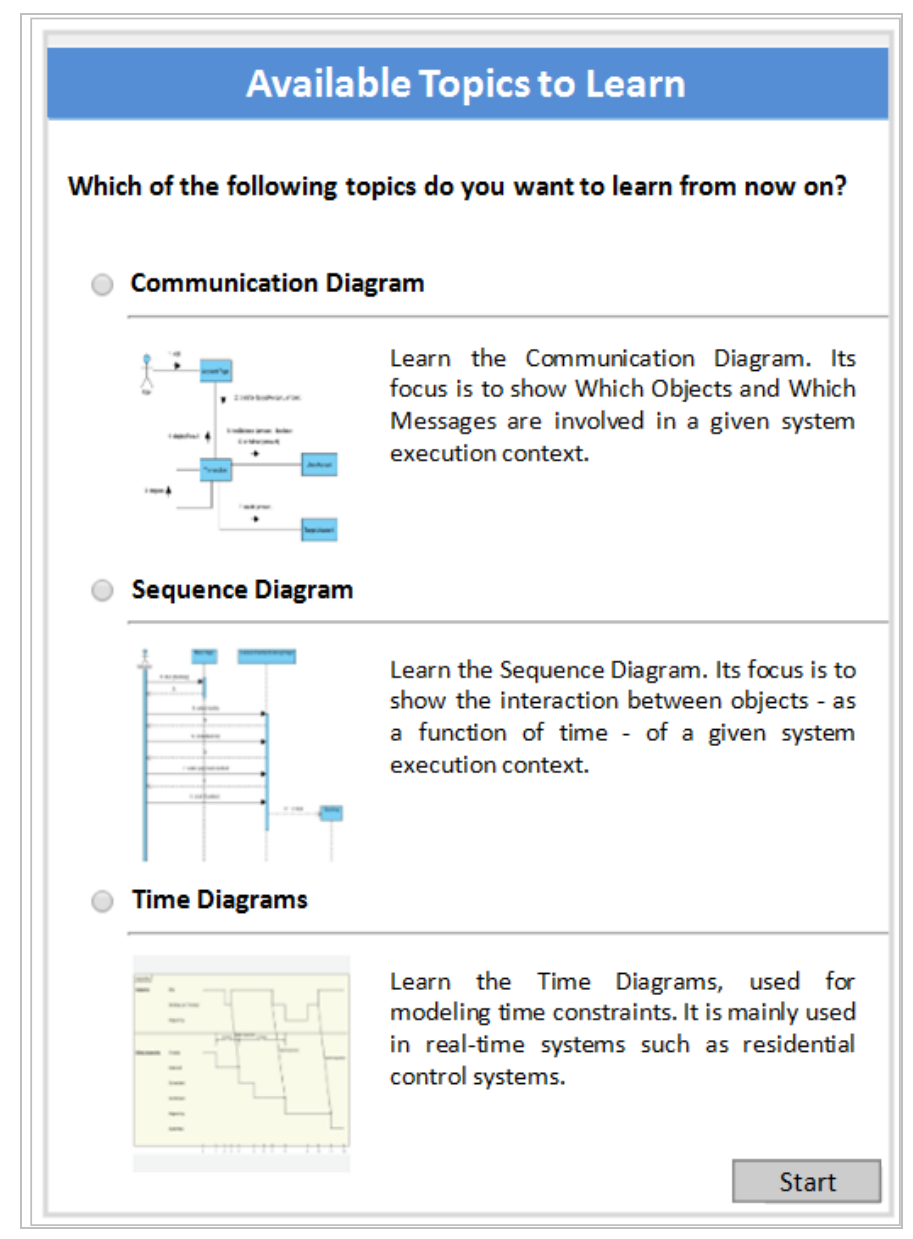

Figure 2: Part of the screen of AdaptWeb where the user chooses the next topic in the course to learn.

In relation to "how much to learn" (from a topic) choice, AdaptWeb allows the user to perform these choices, for instance, when users already know some part of the content of the topic they can skip that part. However, the last version of AdaptWeb doesn't register this kind of choice. The LOs used in AdaptWeb come from different providers; many are "black-boxes", therefore we are not able to register these choices. If LOs could inform clients (e-learning systems) the parts inside them that users used to learn, we would be able to register this choice. In relation to "how to assess learning", there are only multiple-choice online tests in AdaptWeb. Therefore there are no "how to assess learning" options to choose from.

AdaptWeb is linked internally to a LO recommender system that uses the result of the user choices (i.e., the fusion of explicit interactions, learning trace, and contextual information) as a source of information (Section 4.2). The list of LOs presented in Figure 3 is a personalized list of LOs to the user. Therefore, one usage of the learner choice is for learning personalization.

Once the user finishes learning one topic, he/she takes the online test by the link under the list of LOs (Figure 3). Optionally, the user can return to the topics made previously by the "tree of topics” (Figure 3) of the customized program course. When all the topics have been learned, the course ends. 


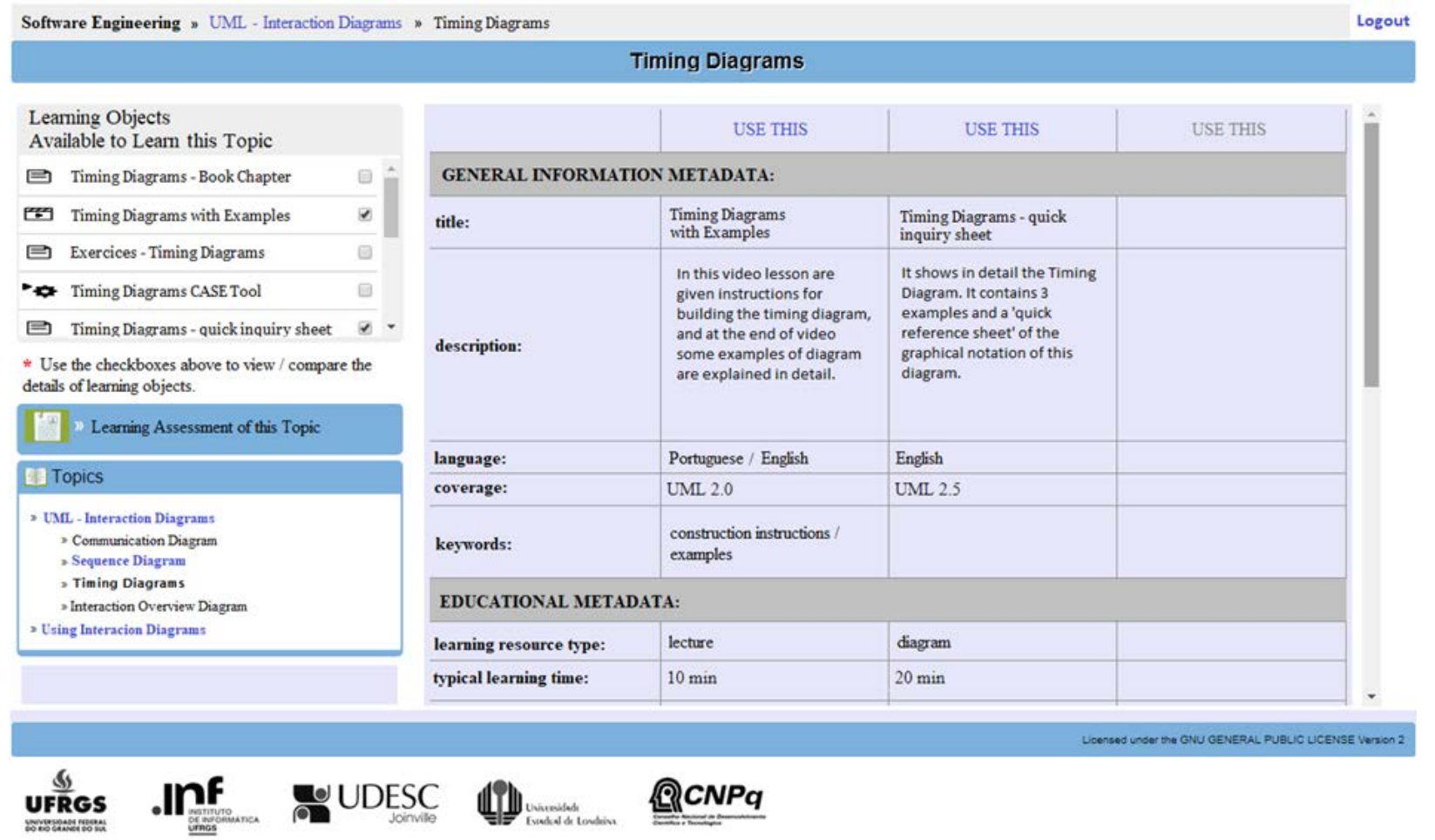

Figure 3: The screen of AdaptWeb where the user performs "how to learn" choices.

\subsection{Usage of the Result of the Learner-User Choices}

As previously mentioned, one usage of the learner choice is for learning personalization. AdaptWeb is linked internally to a recommender system of LOs that uses the result of the user choices as a source of information.

The LO recommendation approach of this recommender system is presented by (Dias \& Wives, 2019). This recommendation approach uses the results of the choices ("how to learn", "with whom to learn", "in which learning pathway to learn", etc.) as implicit feedback. Furthermore, this work presents an experimental evaluation, based on offline and online experiments, which shows that this LO recommendation approach based on the learner-user choices presents higher prediction accuracy than baseline recommendation approaches in the AdaptWeb platform. This result is statistically significant.

\section{Satisfaction Survey with Learner-Users of the AdaptWeb Platform}

The goal of this extended paper is to perform a satisfaction survey with learner-users of the AdaptWeb platform after participating in an online course with the choices from the LearnerDriven Learning paradigm. Satisfaction is the sense of pleasure or disappointment that results from comparing the perceived performance of a product or service against user expectations.

Next, Section 5.1 describes the demographic profile of the research subjects and how the research was built and disseminated. Section 5.2 presents the results and their analysis. 


\subsection{Subjects and Instrument}

In 2018, two classes of students from the Federal University of Rio Grande do Sul attended the online course Interaction Diagrams of UML and responded to a post-course online satisfaction survey. Participation in this survey was anonymous and voluntary.

The questionnaire was developed using Google Forms ${ }^{2}$. The survey was sent to respondents via e-mail at the end of the course. The questionnaire was open for 1 week to be answered. After this the collected data was exported for analysis.

This survey has two open-ended questions (openly ask the opinion): "What did you think about being able to make choices about your learning during this online course?" and "What did you think of the way AdaptWeb makes the choices available to the student?". The advantage of this type of survey questions, over closed-ended questions, is that subjects can respond to the questions exactly as how they would like to answer them, it is, they do not only choose among generic response alternatives (Reja et al., 2003). There were also some demographic questions about age, undergraduate course, and gender.

For each one of the open-ended questions, it was included a word cloud built from the answers of the subjects. A word cloud is a visual form of textual data representation. Each word (simple or compound) has its importance, usually represented by a font size. Therefore one can quickly see the most important terms, and determine their relative importance.

To create a word cloud, initially, the answers of all students were grouped in a text file. The NLTK library was used in Python for stop-word removal in Portuguese. Punctuation and Portuguese accents were also removed, and all words were placed in lower case. Finally, WorldClouds ${ }^{3}$ was used to generate the word cloud.

\subsection{Results and Findings}

Altogether 45 students responded to the survey. The students were from different courses: $64.4 \%$ of the undergraduate course in Computer Science and $35.6 \%$ of the undergraduate course in Computer Engineering. The ages of them ranged from 19 to 29 years old; $15.5 \%$ female, and $84.5 \%$ male.

For each question, all answers were analyzed if it reflected satisfaction and dissatisfaction of the learner-user. Below are the questions, the analysis summary and findings, and some student's comments that that represent the findings.

The first question was: "What did you think about being able to make choices about your learning during this online course?” In short, the students liked enjoyed able to make choices and felt that this possibility was beneficial to their learning. For instance, a student reported "I liked to be able to choose what materials to use to learn each topic in the course ("how to learn" choices), and to choose complementary topics ("what to learn" choices), which show how the subject applies to the area I want to follow in the future. Another student reported "I liked that I was able to choose my learning pathway and my learning pace".

The following are comments of some students and our analysis. About "how to learn" choices, a student reported "the description information of some learning materials (metadata of LOs, in Figure 3) could be more detailed, in my opinion”. We agree with this student; a very

\footnotetext{
${ }^{2}$ https://www.google.com/forms/about/

3 https://www.wordclouds.com/
} 
brief description can lead to poor choices of LOs. Other student reported "the content of some materials is redundant; we have already learned it in other material previously. When a professor chooses the learning material, content does not repeat.” In this student-centered learning paradigm, the student has different ways of learning the same content. The advantage is that he/she can learn the same content from different LOs, for example, in texts, videos, slides, simulators, etc. However, when the student already knows parts of the content completely, he/she has to filter content, skipping what he/she already knows. Although this appears to be a disadvantage of the Learner-driven Learning paradigm, the search and information selection skills need to be developed by the learner for lifelong learning.

Figure 4 shows the world-cloud of this question. The world-cloud was translated into English. The main words are power, harder, ideas, different and options, in this order. It represents the feelings of students when they are allowed to make choices about their learning during this online course. This learning paradigm is different for them, it is harder to choose options of choices, however it generates new ideas, and they are empowered to learn.

The second question was: "What did you think of the way AdaptWeb makes the choices available to the student?" In short, the students liked the way AdaptWeb makes the choices available to students. Most of the students found the system easy to use, intuitive, and the student's choices were explicit and easy to take.

The following are comments of some students, and our analysis. About "what to learn" choices, a student reported "I think the complementary topics should be chosen after finishing the course, because only after learning the basics we do know that a complementary topic will be useful for us." As presented in Section 4.1, in AdaptWeb the "what to learn" choice is taken before starting the learning (after presenting the overview of the course), and it is advised to the student, in case of doubt, ask the teacher for help, to take that choice. Perhaps adding more information about each complementary topic can help the student better in that choice. However, it is an improvement to be analyzed. Other student reported "I chose to do a complementary topic, but during the course I changed my mind, and I could not undo that choice.” In the current version of the AdaptWeb it is not possible to do this, however it is also an improvement to be analyzed.

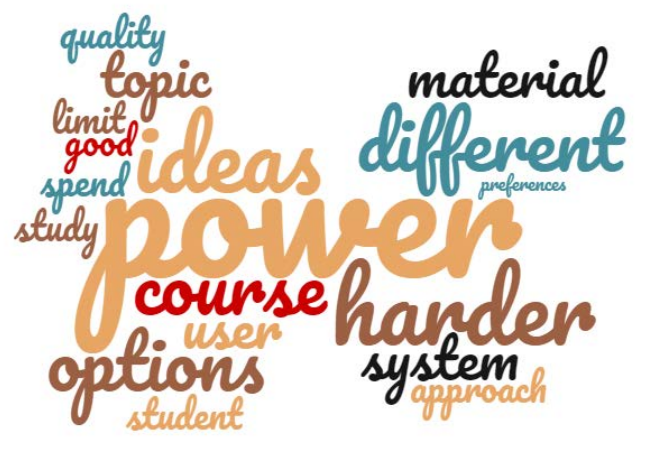

Figure 4: World cloud of question "What did you think about being able to make choices about your learning during this online course?”

Figure 5 shows the world-cloud of this question. The world-cloud was translated into English. The main words are easily, back, nice, click and user, in this order. It represents the feelings of students about the way AdaptWeb makes the choices available to the student. It reflects the interface of the system is easy to use. 


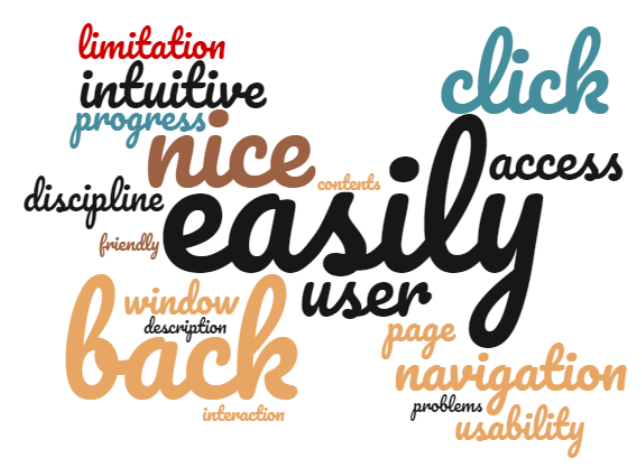

Figure 5: World cloud of question "What did you think of the way AdaptWeb makes the choices available to the student?"

Many students suggested putting a "back button" in order to go back and change past choices. Therefore the word back was very frequent in the answers. Part of these students have pointed to this inability to undo past choices as a limitation or system problem, therefore the words limitation and problems appeared in the cloud, which reflects dissatisfaction.

Summarizing the results of this satisfaction survey, the vast majority of students enjoyed learning in the Learner-driven Learning model, and they liked the way AdaptWeb makes the choices available to students. Most of the students found the system easy to use, intuitive, and the student's choices were explicit and easy to take.

\section{Conclusions}

In this paper, that is an extension of (Dias \& Wives, 2018), we reviewed the definition of the learner choices from the Learner-driven Learning paradigm for e-learning systems. After this, we analyzed how different categories of e-learning systems enable the user to perform such choices, such as MOOCs and PLEs (Personal Learning Environments). In this extended paper, we included in this analysis other categories, such as Online Cooperative Learning Systems, and Serious Games.

Moreover, we extended the Related Work section. As cited, to the best of our knowledge, we were the first to specify the choices from the Learner-driven Learning paradigm of learners for e-learning systems, ensured by the systematic review performed. Therefore there was no way to compare our work "side by side" with existing work. Then we presented intersections between our work and existing work.

We also reviewed the learning process in the AdaptWeb platform and presented, now with more details, how this e-learning platform makes the choices available to learner-users. For illustration, we presented the online course of Interaction Diagrams of UML on the AdaptWeb where the learner-user performs such choices.

We know details about the recommender system of LOs internally linked to AdaptWeb. This recommender system has a LO recommendation approach that uses the result of the learner-user choices as implicit feedback. (Dias \& Wives, 2019) presents this LO recommendation approach, and an experimental evaluation which shows that it presents higher prediction accuracy than baseline recommendation approaches in the AdaptWeb. This result is statistically significant. 
The goal of this extended paper was to present the satisfaction survey performed with two classes of students after the online course of Interaction Diagrams of UML on the AdaptWeb platform. The survey questions were about making choices during learning and about the way AdaptWeb makes the choices available to learner-users. Summarizing the results, students liked enjoyed able to make choices about their own learning and felt that this possibility was beneficial to their learning. And they liked the way AdaptWeb makes the choices available to students. Most of the students found the system easy to use, intuitive, and the student's choices were explicit and easy to take.

\section{Acknowledgments}

This study was financed in part by the Coordenação de Aperfeiçoamento de Pessoal de Nível Superior - Brasil (CAPES) - Finance Code 001.

\section{References}

Alexander, S., Kernohan, G., \& Mccullagh, P. (2004) Self-directed and lifelong learning. Global Health Informatics Education, 109, 152-66.

Dias, S. A., \& Wives, L. K. (2018). Definition of Learner Choices from Learner-Driven Learning for Ubiquitous e-Learning Systems and its Application in the AdaptWeb Platform. Brazilian Symposium on Computers in Education (Simpósio Brasileiro de Informática na Educação-SBIE), v. 29, n. 1, p. 118. doi:http://dx.doi.org/10.5753/cbie.sbie.2018.118 [GS Search]

Dias, S. A., \& Wives, L. K. (2018-b). Assessment of the Most Relevant Learning Object Metadata. Proceedings of the 10th International Conference on Computer Supported Education (CSEDU 2018), p. 175-182. doi:http://dx.doi.org/10.5220/0006660601750182 [GS Search]

Dias, S. A., \& Wives, L. K. (2019). Recommender system for learning objects based in the fusion of social signals, interests, and preferences of learner users in ubiquitous e-learning systems. Personal and Ubiquitous Computing, 23(2), 249-268. [GS Search] doi: https://link.springer.com/article/10.1007/s00779-018-01197-7

Downes, S. (2008). Connectivism: A Theory of Personal Learning. Retrieved from http://www.slideshare.net/Downes/connectivism-a-theory-of-personal-learning

Drachsler, H., Verbert, K., Santos, O., \& Manouselis, N. (2015) Panorama of Recommender Systems to Support Learning. In: Ricci F., Rokach L., Shapira B. (eds) Recommender Systems Handbook (pp.421-451) Boston, MA: Springer.

Gates-Dell-EDUCAUSE - Bill \& Melinda Gates Foundation, the Michael and Susan Dell Foundation, and EDUCAUSE (2017) A working definition of personalized learning. Retrieved from https://www.documentcloud.org/documents/1311874-personalized-learning -working-definition-fall2014.html

Ginsberg, M. B. (2015) Excited to Learn: Motivation and Culturally Responsive Teaching. Corwin Press.

iNACOL - International Association for K-12 Online Learning; Patrick, S. Kennedy, K. Powell, A (2013) Mean What You Say: Defining and Integrating Personalized, Blended and Competency Education. Retrieved from: https://www.inacol.org/resource/mean-what-yousay-defining-and-integrating-personalized-blended-and-competency-education/ 
Intayoad, W., Kamyod, C., \& Temdee, P. (2020). Reinforcement Learning Based on Contextual Bandits for Personalized Online Learning Recommendation Systems. Wireless Personal Communications, 1-16. doi: https://doi.org/10.1007/s11277-020-07199-0

LEADLAB - The LEADLAB Project (2010) Retrieved from: http://leadlab.euproject.org/ services/files/Download/LEADLABMODEL-EN.pdf

Manouselis, N., Vuorikari, R., \& Assche, F. V. (2010) Collaborative recommendation of e-learning resources: an experimental investigation. Journal of Computer Assisted Learning, 26(4), 227-242. doi:https://doi.org/10.1111/j.1365-2729.2010.00362.x

Mackness, J., Sui, M., \& Roy, W. (2010) The ideals and reality of participating in a MOOC. Proceedings of the $7^{\text {th }}$ International Conference on Networked Learning. University of Lancaster. [GS Search]

McClaskey, K. (2016). Developing the expert learner through the stages of personalized learning. Retrieved December, 21, 2018. [GS Search]

Miliband, D. (2006) Choice and voice in personalized learning. In: OECD, Personalizing education. doi:http://dx.doi.org/10.1787/9789264036604-2-en

Passe, J. (1996) When students choose content: A guide to increasing motivation, autonomy, and achievement. Washington, DC: National Academies Press.

Reja, U., Manfreda, K. L., Hlebec, V., \& Vehovar, V. (2003) Open-ended vs. Close-ended Questions in Web Questionnaires, Developments in Applied Statistics, 19, 159-77. [GS Search]

Song, Y. (2017) Personalized Learning on MOOCs. MS thesis. University of Lapland, Finland. Retrieved from: https://www.semanticscholar.org/paper/Personalised-Learning-on-MOOC s-Song/302f42c932118afe780b31c9d4041fc440358a9f

Watkins, C., Carnell, E. \& Lodge, C. (2007) Effective Learning in Classrooms. London, Paul Chapman Publishing. doi:http://dx.doi.org/10.4135/9781446211472

Zheng, Y., Mobasher, B. \& Burke, R. (2014) Context Recommendation Using Multi-label Classification. Proceedings of the 2014 IEEE/WIC/ACM International Joint Conferences on Web Intelligence (WI) and Intelligent Agent Technologies (IAT). doi: https://doi.org/10.1109/WI-IAT.2014.110

Zhuhadar, L., \& Butterfield, J. (2014) Analyzing Students Logs in Open Online Courses Using SNA Techniques. Proceedings of the Americas Conference on Information Systems, AMCIS, Savannah, Georgia, USA. 